\section{OP0158-HPR TELEMEDICINE CONSULTATIONS IN POLYMYALGIA RHEUMATICA PATIENTS. A TWO YEARS' EXPERIENCE REPORT}

\author{
S. Fredslund-Andersen ${ }^{1}$, P. Lage-Hansen ${ }^{1}$, N. Svendsen ${ }^{1}$, M. Jeppesen \\ Rechnagel $^{1}$, U. Højberg ${ }^{1}$, N. J. Kock ${ }^{1}$, J. Krause Sørensen ${ }^{1}$, S. Chrysidis ${ }^{1}$. \\ ${ }^{1}$ Hospital of Southwest Jutland, University of Southern Denmark, Esbjerg, \\ Denmark
}

Background: Polymyalgia rheumatica (PMR) is the most common inflammatory rheumatic disease of people over 50 years in Scandinavian countries and people of northern European descent, characterized by proximal pain, stiffness, raised inflammatory markers and prompt response to steroids(1). Relapse free during tapering off prednisone, are common in approximately $50 \%$ of patients. Studies reported that up to $70 \%$ of PMR patients are successfully tapered of prednisone due to remission, within 1-2 years from treatment initiation (1). Telemedicine has found a wider application in a number of chronic diseases resulting in a decreased number of hospital visits; however, no telemedicine studies on PMR patients have been performed before (2).

Objectives: To evaluate the use of telemedicine consultation in newly diagnosed PMR patients.

Methods: Telemedicine consultations managed by rheumatic nurses for newly diagnosed PMR patients was established in 2017 at our department. Patients diagnosed with PMR by a rheumatologist received written information concerning the nature of the disease, a "follow-up" schedule and a prednisone treatment/ tapering plan. Telephone consultations (TC) including relevant blood analysis was planned after 4, 16, 52 and 78 weeks from the time of diagnosis. TC was managed by 4 nurses specialized in rheumatic diseases, who received appropriate education of PMR prior to the establishment. A predefined questionnaire was used for every telephone consultation.

All PMR patients were initially treated with $15 \mathrm{mg}$ of prednisone daily, with slowly tapering to $5 \mathrm{mg}$ daily at week 16 and reduced to zero at week 48 . In the case of relapse symptoms, patients were instructed to contact the treating nurses. In that case, relevant biomarkers were taken and a rheumatologist evaluated the need for a physical consultation and potential treatment adjustment.

Only patients with minimum disease duration of 3 months were included in the study. Results: In a period of two years, 76 patients were evaluated. The mean age was 73 years and the mean follow up period was 10,67 \pm standard deviation (SD) 5,2 months. At the time of diagnosis, all patients fulfilled the 2012 Classifications criteria for PMR (3). The Mean number of TC was 4.27( \pm SD 2, 3).

In 45 cases $(60 \%)$ no additional physical consultation was necessary. In patients examined physically due to relapse suspicion, the most common findings were PMR relapses $(66 \%)$, followed by non-inflammatory muscle and joint pain $(18,5 \%)$, arthritis $(14 \%)$, while one patient was diagnosed with giant cell arteritis.

Successfully prednisone tapering was achieved in 23 cases (30\%) while 27 patients $(35,5 \%)$ at the time of data evaluation were treated with only $2,5 \mathrm{mg}$ of prednisone daily. The mean current daily prednisolone dosage was $3.95 \mathrm{mg}( \pm$ SD 3.25) while disease-modifying-anti-rheumatic-drugs had been initiated in 6 patients.

Conclusion: Telemedicine consultations in PMR diminish the need for physical consultations in this patient cohort. More than half of all patients were either out of- or received only a very low dose of prednisone at the time of evaluation of data.

References:

[1] Kermani TA, Warrington KJ. Polymyalgia rheumatica. Lancet. 2013 Jan 5;381(9860):63-72. doi: 10.1016/S0140-6736(12)60680-1.

[2] Matteo Piga et al. Telemedicine for patients with rheumatic diseases: Systematic review and proposal for research agenda. Semin Arthritis Rheum. 2017 Aug;47(1):121-128. doi: 10.1016/j.semarthrit.2017.03.014. Epub 2017 Mar 22.

[3] Dasgupta B, et al. 2012 Provisional classification criteria for polymyalgia rheumatica: A European League Against Rheumatism/American College of Rheumatology collaborative initiative. Arthritis Rheum. 2012; 64: 943-54.

Disclosure of Interests: None declared

DOI: 10.1136/annrheumdis-2020-eular.3261

\section{OP0159 \\ EFFICACY OF COMPREHENSIVE TECHNOLOGY- ASSISTED HOME-BASED EXERCISE IN ANKYLOSING SPONDYLITIS: A RANDOMIZED, CONTROLLED TRIAL}

Y. Wang ${ }^{1}$, X. $\mathrm{Liu}^{2}$, Y. Shi ${ }^{3}$, X. Ji ${ }^{1}$, W. Wang ${ }^{3}$, W. Jiao ${ }^{2}$, F. Huang ${ }^{1} .{ }^{1}$ Chinese PLA General Hospital, Department of Rheumatology, First Medical Center, Beijing, China; ${ }^{2}$ Beijing Sport University, College of Sport Medicine and Sport Rehabilitation, Beijing, China; ${ }^{3}$ Chinese PLA General Hospital, Health Management Institute, Second Medical Center, Beijing, China

Background: Clinical practice guidelines recommend that exercise is an essential component in the self-management of Ankylosing Spondylitis (AS).
Attending supervised interventions requiring periodic medical center visits can be burdensome and patients may decline participation, whereas, effective home-based exercise interventions that do not need regular medical center visits are likely to be more accessible and acceptable for patients with AS. Recently, increasing evidences have been accumulated that the wearable devices could facilitate patients with inflammatory arthritis by giving exercise instructions and improving self-efficacy. Therefore, patients with AS may benefit from an effective technology-assisted home-based exercise intervention.

Objectives: To investigate the efficacy of a comprehensive technology-assisted home-based exercise intervention on disease activity in patients with AS.

Methods: This study was a 16-week assessor-blinded, randomized, waiting-list controlled trial (ChiCTR1900024244). Patients with AS were randomly allocated to the home-based exercise intervention group and the waiting-list control group A 16-week comprehensive exercise program consisting of a moderate intensity (64\%-76\% HR ${ }_{\max }$ ) aerobic training for 30 min on 5 days/week and a functional training for $60 \mathrm{~min}$ on 3 days/week was given to patients in the intervention group immediately after randomization, with $1.5 \mathrm{~h}$ training sessions for two consecutive days by a study physical therapist at baseline and Week 8 . The aerobic exercise intensity was controlled by a Mio FUSE Wristband with a smartphone application. The functional training consisted of the posture training, range of motion exercises, strength training, stability training and stretching exercises. Patients in control group received standard care during the 16-week follow-up and started to receive the exercise program at Week 16. The primary outcome was ASDAS at Week 16. The secondary outcomes were BASDAI, BASFI, BASMI, ASAS HI, peak oxygen uptake, body composition and muscle endurance tests. The mean difference between groups in change from baseline was analyzed with the analysis of covariance.

Results: A total of 54 patients with AS were enrolled ( 26 in intervention group and 28 in control group) and $46(85.2 \%)$ patients completed the 16-week follow-up. The mean difference of ASDAS between groups in change from baseline to 16 -week follow-up was $-0.2(95 \% \mathrm{Cl},-0.4$ to $0.003, \mathrm{P}=0.032)$ and the mean change from baseline was $-0.4(95 \% \mathrm{Cl},-0.5$ to -0.2$)$ in the intervention group vs $-0.1(95 \% \mathrm{Cl},-0.3$ to 0.01$)$ in the control group, respectively. Significant between-group differences were found between groups for BASDAI $(-0.5[95 \% \mathrm{Cl},-0.9$ to -0.2$], \mathrm{P}=0.004)$, BASMI $(-0.7[95 \% \mathrm{Cl}$ -1.1 to -0.4 ], $\mathrm{P}<0.001)$, BASFI $(-0.3$ [95\% $\mathrm{Cl},-0.6$ to 0.01 ], $\mathrm{P}=0.035)$, peak oxygen uptake $(2.7[95 \% \mathrm{Cl}, 0.02$ to 5.3$] \mathrm{ml} / \mathrm{kg} / \mathrm{min}, \mathrm{P}=0.048)$ and extensor endurance test $(17.8[95 \% \mathrm{Cl}, 0.5$ to 35.2$] \mathrm{s}, \mathrm{P}=0.044)$ at Week 16 . Betweengroup differences were detected in ASAS $\mathrm{HI}(-0.9[95 \% \mathrm{Cl},-1.7$ to -0.1$]$ $\mathrm{P}=0.030)$, body fat percentage $(-1.0[95 \% \mathrm{Cl},-2.0$ to -0.01$] \%, \mathrm{P}=0.048)$ and visceral adipose tissue $\left(-4.9[95 \% \mathrm{Cl},-8.5\right.$ to -1.4$\left.] \mathrm{cm}^{2}, \mathrm{P}=0.008\right)$ at Week 8 , but not at Week 16. No significant between-group differences were detected in the total lean mass, time up and go test and the flexor endurance test during the follow-up.

Conclusion: Comprehensive technology-assisted home-based exercise has been shown to have beneficial effects on disease activity, physical function, spinal mobility, aerobic capacity, and body composition as well as in improving fatigue and morning stiffness of patients with AS.

References:

[1] van der Heijde D, Ramiro S, Landewé R, et al. Ann Rheum Dis 2017;76:978-991.

Disclosure of Interests: None declared

DOI: 10.1136/annrheumdis-2020-eular.5100

\section{Advances in treating SLE and lupus nephritis}

\section{\begin{tabular}{|l|l|l|l|}
\hline OP0160 & HYDROXYCHLOROQUINE BLOOD LEVELS AND RISK
\end{tabular} OF THROMBOTIC EVENTS IN SYSTEMIC LUPUS ERYTHEMATOSUS}

M. A. Petri ${ }^{1}$, M. Konig ${ }^{1}$, J. LI ${ }^{1}$, D. Goldman ${ }^{1} .{ }^{1}$ Johns Hopkins University, Medicine/Rheumatology, Baltimore, United States of America

Background: Hydroxychloroquine (HCQ) has a primary role in the treatment of systemic lupus erythematous (SLE). Beyond its pleiotropic immunomodulatory effects on Toll-like receptor and type I interferon signaling, HCQ use has been found to be protective for thrombosis in SLE (1). Optimal dosing of HCQ in SLE is unknown. The longitudinal measurement of HCQ blood levels may provide an opportunity to individualize weight-based dosing strategies and reduce risk of toxicity.

Objectives: Examine the association of HCQ blood levels with thrombotic events in a longitudinal SLE cohort.

Methods: 812 SLE patients with HCQ blood level measured prior to the thrombosis were included: $93 \%$ female, $43 \%$ African-American, $46 \%$ Caucasian. $\mathrm{HCQ}$ blood levels were quantified by liquid chromatography-tandem mass 
spectrometry. Mean HCQ blood levels ( \pm standard deviation) over all cohort visits prior to occurrence of thrombosis were calculated for each patient. Thromboses were defined as venous (DVT/PE or other venous) or arterial thrombosis (stroke, myocardial infarction, digital gangrene or other arterial).

Results: Thrombosis had occurred during prospective follow up in 43 patients (5.5\%), venous in $3.0 \%$ and arterial in $2.9 \%$. Lupus anticoagulant was strongly associated with a history of any thrombosis (OR $3.25, p<0.0001$ ), venous thrombosis (OR 3.53, $\mathrm{p}<0.0001$ ), and arterial thrombosis (OR 3.08, $\mathrm{p}<0.0001$ ). A prospective analysis shows that for any thrombosis and for venous thrombosis, the HCQ blood level was significantly lower (Table 1). Higher prescribed doses of $\mathrm{HCQ}$ (as opposed to HCQ blood levels) were also associated with decreased odds of any thrombosis and venous thrombosis in a separate cross-sectional analysis (OR $0.88, p=0.04$ and OR $0.83, p=0.009$, respectively for each $1 \mathrm{mg} / \mathrm{kg}$ increase in prescribed $\mathrm{HCQ}$ ).

Table 1. Thrombotic Events are Associated with Lower Mean HCQ Blood Level

\begin{tabular}{lccc}
\hline & \multicolumn{2}{c}{ Mean HCQ Blood Level $( \pm$ Std. Dev. $)$} & \\
\cline { 2 - 3 } & Thrombotic Event & No Event & P-value \\
\hline Any thrombosis & $695 \pm 464$ & $887 \pm 562$ & 0.029 \\
Any venous thrombosis & $682 \pm 374$ & $881 \pm 560$ & 0.10 \\
DVT/PE only & $615 \pm 384$ & $881 \pm 559$ & 0.055 \\
Any arterial thrombosis & $708 \pm 539$ & $882 \pm 558$ & 0.13 \\
Stroke & $720 \pm 643$ & $880 \pm 557$ & 0.27 \\
\hline
\end{tabular}

Conclusion: $\mathrm{HCQ}$ blood levels are inversely associated with risk of any thrombosis and of venous thrombosis in patients with SLE in a prospective analysis. Reduction of HCQ dosing, as suggested by the American Academy of Ophthalmologists (2), could reduce or eliminate the benefit of hydroxychloroquine to prevent thrombosis

References:

[1] Petri M. Use of hydroxychloroquine to prevent thrombosis in systemic lupus erythematosus and in antiphospholipid antibody-positive patients. Curr Rheumatol Rep 2011;13:77-80.

[2] Marmor MF, Kellner U, Lai TYY, Melles RB, Mieler WF. Recommendations on screening for chloroquine and hydroxychloroquine retinopathy (2016 revision). Ophthalmology 2016;123:1386-1394.

Acknowledgments: The Hopkins Lupus Cohort is supported by NIH Grant RO1 AR069572

Disclosure of Interests: Michelle A Petri Grant/research support from: GSK, Eli Lilly and Company, Consultant of: Eli Lilly and Company, Maximilian Konig: None declared, Jessica Li: None declared, Daniel Goldman: None declared

DOI: 10.1136/annrheumdis-2020-eular.1236

\section{OP0161 ASSOCIATION OF BASELINE CYTOTOXIC GENE EXPRESSION WITH USTEKINUMAB RESPONSE IN SYSTEMIC LUPUS ERYTHEMATOSUS}

L. Seridi ${ }^{1}$, M. Cesaroni ${ }^{1}$, M. J. Loza ${ }^{1}$, J. Schreiter ${ }^{1}$, K. Sweet $^{1}$, Y. Orlovsky ${ }^{1}$, I. Baribaud ${ }^{1}$, A. Orillion ${ }^{1}$, P. Lipsky ${ }^{2}$, R. V. Vollenhoven ${ }^{3}$, B. H. Hahn ${ }^{4}$, G. Tsokos ${ }^{5}$, M. Chevrier ${ }^{1}$, S. Rose ${ }^{1}$, F. Baribaud ${ }^{1}$, J. Jordan ${ }^{1} .{ }^{1}$ Janssen Research \& Development, LLC, Spring House, PA, United States of America; ${ }^{2}$ AMPEL BioSolutions, LLC, Charlottesville, VA, United States of America; ${ }^{3}$ Amsterdam Rheumatology and Immunology Center, Amsterdam, Netherlands; ${ }^{4}$ University of California, Los Angeles, Los Angeles, CA, United States of America; ${ }^{5}$ Beth Israel Deaconess Medical Center, Harvard Medical School, Boston, MA, United States of America

Background: Systemic Lupus Erythematous (SLE) is a clinically and biologically diverse disease, for which only one new therapy has been approved in the past 60 years. In a phase 2 trial on patients with mild-to-moderate SLE, ustekinumab (UST) improved clinical and laboratory measures of disease activity compared with placebo (PBO). ${ }^{1}$

Objectives: We previously reported an association of IFN- $\gamma$ reduction with response to UST, ${ }^{2}$ suggesting an impact on the IL12/Th1 axis. To extend these findings, we performed unbiased transcriptomic analysis from baseline whole blood samples to identify genes that discriminate UST responders (UST-R) from non-responders (UST-NR) using the primary endpoint of Systemic Lupus Erythematosus Responder Index (SRI)-4 at week 24 to define response.

Methods: UST was studied in a Ph2 PBO-controlled study of 102 patients with seropositive SLE and active disease despite standard therapy. Patients were randomized $3: 2$ to receive IV UST $6 \mathrm{mg} / \mathrm{kg}$ or placebo followed by subcutaneous injections of UST $90 \mathrm{mg}$ or PBO every 8 weeks. Whole blood gene expression at baseline was measured via microarray using RNA samples from 100 patients, as samples from 2 patients failed quality control. An unbiased approach was used to identify gene signatures present at baseline that associate with UST response. Recombinant IL-12 or IL-23 was incubated in vitro with whole blood from 6 healthy donors for $24 \mathrm{~h}$ and RNA-Seq was performed to determine the effect of these treatments on representative genes comprising the UST response signature.

Results: A non-biased machine learning algorithm identified a 9-gene whole blood signature composed primarily of cytotoxic cell-associated transcripts (PRF1, KLRD1, GZMH, NKG7, GNLY, FGFBP2, TRGC2, TARP, TRGV2) that was enriched at baseline in UST-R vs UST-NR. By Gene Set Variation Analysis, the cytotoxic signature enrichment in UST-NR was less at baseline than both UST-R and a healthy control cohort $(P=0.0087, P=0.056$, respectively), whereas UST-R cytotoxic gene enrichment was similar to healthy controls $(P=0.31)$. No significant difference in cytotoxic signature enrichment was observed at baseline between $\mathrm{PBO}$ responders and PBO non-responders or healthy controls (Figure). Enrichment levels of the cytotoxic gene signature remained stable over time in PBO and UST-NR groups while a trend of decreased cytotoxic signature was observed in UST-R, although never reaching levels seen in UST-NR. To begin to understand the relationship between $\mathrm{IL}-12$ and IL-23, the targets of UST, and the cytotoxic signature, whole blood was stimulated with these cytokines in vitro. Recombinant IL-12, but not IL-23 resulted in increased expression of representative members of this cytotoxic gene signature.

Conclusion: We identified a novel cytotoxic signature in baseline blood samples that associated with UST response in SLE. The observation that IL-12 can increase this signature in vitro and that IL-12 is a robust inducer of cytotoxic cel activity $^{3}$ as well as IFN- $\gamma^{3}$ suggests an important role of IL-12 blockade in the mechanism of action of UST in SLE.

References:

[1] van Vollenhoven RF. Lancet. 2018;392:1330-39

[2] Jordan. ACR 2018 Abstract \# 2951

[3] G. Trinchieri. Nat Rev Immunol. 2003;3:133-46

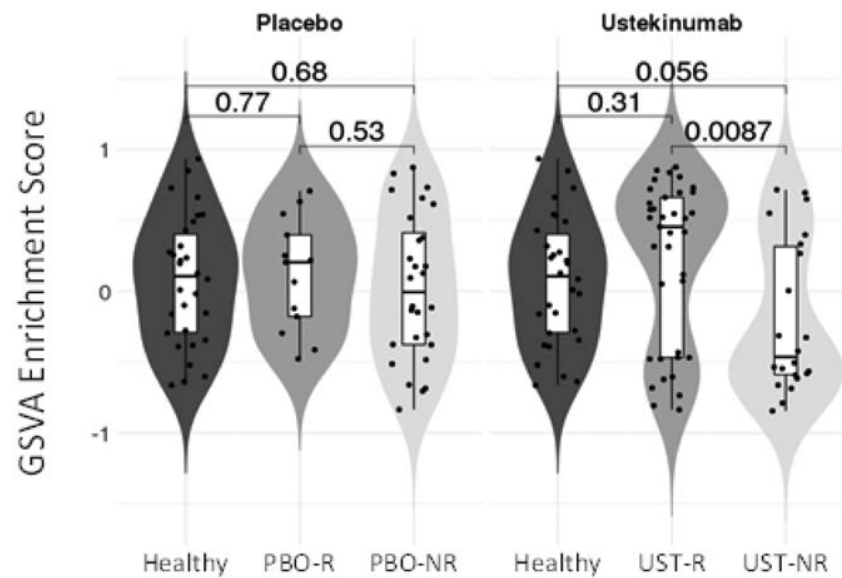

Figure.

Disclosure of Interests: Loqmane Seridi Employee of: Janssen Research \& Development, LLC, Matteo Cesaroni Employee of: Janssen Research \& Development, LLC, Matthew J Loza Employee of: Janssen Research \& Development LLC, Jessica Schreiter Employee of: Janssen Research \& Development, LLC Kristen Sweet Employee of: Janssen Research \& Development, LLC, Yevgeniya Orlovsky Employee of: Janssen Research \& Development, LLC, Spring House PA, United States of America, Isabelle Baribaud Employee of: Janssen Research \& Development, LLC, Ashley Orillion Employee of: Janssen Research \& Development, LLC, Peter Lipsky Consultant of: Horizon Therapeutics, Ronald van Vollenhoven Grant/research support from: AbbVie, Amgen, Arthrogen, Bristol-Myers Squibb, GlaxoSmithKline (GSK), Janssen Research \& Development, LLC, Lilly, Pfizer, Roche, and UCB, Consultant of: AbbVie, AstraZeneca, Biotest, Bristol-Myers Squibb, Celgene, Crescendo Bioscience, GSK, Janssen, Lilly, Medac, Merck, Novartis, Pfizer, Roche, UCB and Vertex, Speakers bureau: AbbVie AstraZeneca, Biotest, Bristol-Myers Squibb, Celgene, Crescendo Bioscience, GlaxoSmithKline, Janssen, Lilly, Merck, Novartis, Pfizer, Roche, UCB, Vertex Bevra H. Hahn Grant/research support from: Janssen Research \& Development, LLC, George Tsokos Grant/research support from: Janssen Research \& Development, LLC, Marc Chevrier Employee of: Janssen Research \& Development, 